\title{
Emergency Smartwatch for Fisherman
}

\author{
Saoud Hamidou, Mohamed Abdallah Abdoul-Halim, Rawad Abdulghafor, Sharyar Wani \\ Dept. of Computer Science, Faculty of Information and Communication Technology, International Islamic University Malaysia, Kuala Lumpur, Malaysia \\ saoudhamad9@gmail.com,mohamedabdallah592@gmail.com, rawad@iium.edu.my,sharyarwan@iium.edu.my
}

\begin{abstract}
This work is about designing a smartwatch that is used mostly by fishermen. The watch provides the location of the user using the global positioning system (GPS) and a panic button that he can use in case of emergency. It can record the heart rate by using a heart rate sensor. The paper works with a software that is used by the appropriate authorities; they record the information provided by the smartwatch. The third part of the paper is a mobile application used by the rescue team; the application shows the location of the fisherman. This paper aims to help fishermen in case of emergency cases, they can be saved by the rescue team. Based on the heart rate sensor and the panic button provided by the smartwatch, the appropriate authorities can know when a fisherman needs help and send the rescue team.
\end{abstract}

Keywords - Emergency Smartwatch, Heartbeat Rate, Fisherman, Rescue Team.

\section{INTRODUCTION}

Since the apparition of human beings, many activities have been invented in order to sustain themselves with food and nutriments. Among those activities we can cite "Fishing". From the antiquity, fishing has been a prolific commercial activity [1]. The common technique used by fishermen, is to go to the sea using a boat and capture the fishes. This project is composed by a hardware and software. The hardware is the smartwatch that the fishermen will put on their wrist and the software works as a database for the smartwatch users. It collects the heart rate of the user; his location using the global positioning system (GPS) and provide a panic button to be used in case of emergency. As it is not useful to use handphone in sea, the smartwatch will not rely on handphone and it will be directly connected to the sever.

In addition, the data collected such as location and heartbeat rate will be transferred in our server through satellite or electromagnetic spectrum waves. From the period of antiquity to the modern era, Fishing has been a great source of income for many countries. Nowadays, this activity is mostly done by using modern techniques. However, in many countries, especially third world countries, fishing is done individually and the techniques used are still archaic. Furthermore, some people don't go for fishing for business purposes, but they fish as a fun activity. It has been noticed that going to fish individually or in pair can be dangerous because the person is exposed to a serious danger which is getting lost. In fact, people get lost in the sea frequently and it is very hard for them to survive and also very hard for the authorities to find them once they get lost. In that sense, our project aims to ameliorate the conditions of security that will ease their work and make them feel more secure when doing their job

\section{RELATED WORK}

In the work of [2], a smartwatch has designed that includes hardware and software necessary to obtain motion and sensor data from a user wearing the smartwatch. The smartwatch is be able to collect useful data. The smartwatch has many important components which are:

- A motion sensor to sense motion data.

- Biometric sensor to sense biometric data such as fingerprint, Iris recognition or facial recognition.

However, there are some weaknesses about the system because some important features are missing such as GPS, heart rate monitor and Panic button.

In [3], an invention has been created and it can use not only to measure physiological parameters, such as body temperature, pulse and blood pressure of a human body, but also to monitor a motion state, particularly behaviours like falling down, of the human body. It also has a positioning system and a function of calling emergency numbers. This project is very useful in terms of health and security conditions because it tracks the whole human body and also provide a location in case of emergency. However, gathering all these information and functionalities makes the wristwatch very complex to use and not all users will be able to use it properly.

In [4], A wearable device for personal safety has was built. The client connects the wearable device with an application and it detects all the cases of emergency that are happening within a close range to the user. The wearable device also 
provides the nature of the emergency so that the user will be able to prepare himself to face that emergency.

The strength of this invention is that it prevents the user from a potential danger. However, it is not really helping the person who is directly facing the emergency. It doesn't tell the user about his health and it is not connected to a rescue team. So, this invention is only based on prevention.

In [5], a signalling device which called "tracking and safety smartwatch" that is worn in the manner of an accessory. The invention communicates through messages with an appropriate authority so the users wearing the smartwatch can be monitored. The "tracking and safety smartwatch" allows the appropriate authority to determine the location of the users. The device provides for the transmission of an emergency message from the individual to the appropriate authority. Basically, the invention emphasizes on providing the exact location of the user and provides an emergency button for urgent cases but when the user loses his consciousness, it is impossible for the appropriate authority to know that the user needs help, because the device doesn't have any heart rate monitoring system.

\section{Methodology}

\section{A. Implementation}

The human heart is an organ that pumps blood throughout the body via the circulatory system, supplying oxygen and nutrients to the tissues and removing carbon dioxide and other wastes. The cardiac cycle is the sequence of events that occurs when the heart beats. As the heartbeats, it circulates blood through pulmonary and systemic circuits of the body [6].

The human heart is an organ that pumps blood throughout the body via the circulatory system, supplying oxygen and nutrients to the tissues and removing carbon dioxide and other wastes. The cardiac cycle is the sequence of events that occurs when the heart beats. As the heartbeats, it circulates blood through pulmonary and systemic circuits of the body [6].

The heart rate is the number of times the heartbeats in the space of a minute. In order to know if the heart is working correctly is to use a heart rate monitor. and based on the results, we can know whether the heart is functioning normally or not.

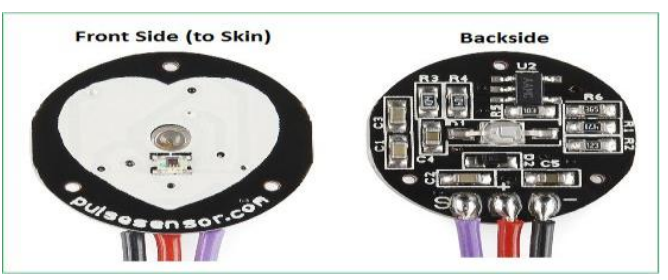

Fig. 1 Heart rate sensor from Arduino

\section{Global Positioning System (GPS)}

The Global Positioning System (GPS) is a space-based satellite navigation system that provides location and time information in all weather conditions, anywhere on or near the Earth where there is an unobstructed line of sight to four or more GPS satellites. The system provides critical capabilities to military, civil and commercial users around the world. It is maintained by the United States government and is freely accessible to anyone with a GPS receiver.

Global Positioning System tracking is a method of working out exactly where something is. A GPS tracking system, for example, may be placed in a vehicle, on a cell phone, or on special GPS devices, which can either be a fixed or portable unit. GPS works by providing information on exact location. It can also track the movement of a vehicle or person. So, for example, a GPS tracking system can be used by a company to monitor the route and progress of a delivery truck, and by parents to check on the location of their child, or even to monitor high-valued assets in transit [7].

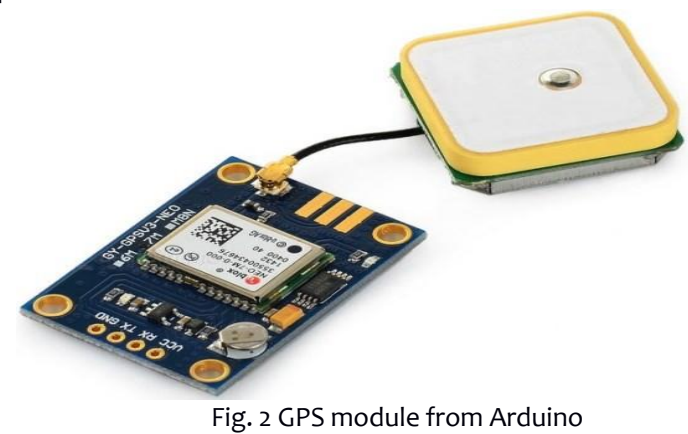

III. PANIC BUTTON SOS BUTTON

A panic button is an electronic device designed to alert public safety officials in an emergency situation where there is an immediate threat. In the past, several different kinds of panic buttons have emerged as popular security tools, and each notifies the proper first response team or local law enforcement in different ways. There's a panic button alarm, which might be installed in a car, house, or other property. When the alarm is triggered, it sends off a loud noise in addition to notifying the affiliated alarm company [8].

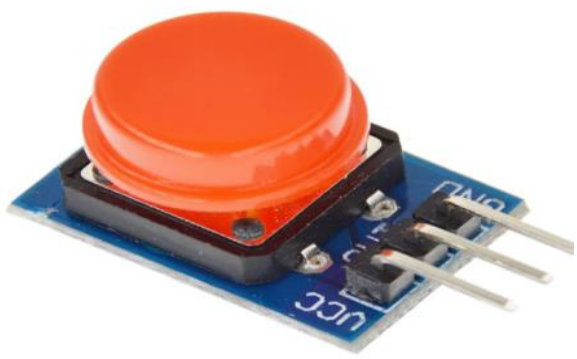

Figure 3: Push Button from Arduino 


\section{ESP8266}

"ESP8266" is A Wi-Fi module that connects the mobile that connects the prototype of the hardware to the mobile application that we designed. It can be connected to the computer by using a USB cable, the program is uploaded and stored on the chip. The information recorded by the hardware are sent to the mobile application through the module.

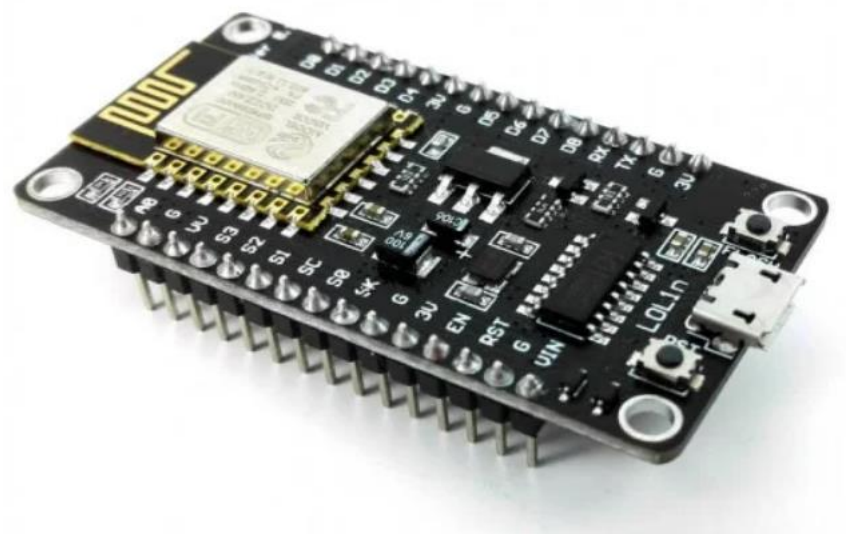

Fig. 4 NodeMCU LUA V3 ESP8266 WI-FI

\section{Breadboard}

Breadboard is a component used to build electronic devices without using a soldering iron. Components are pushed into the sockets on the breadboard and the extra wires are used to make connections

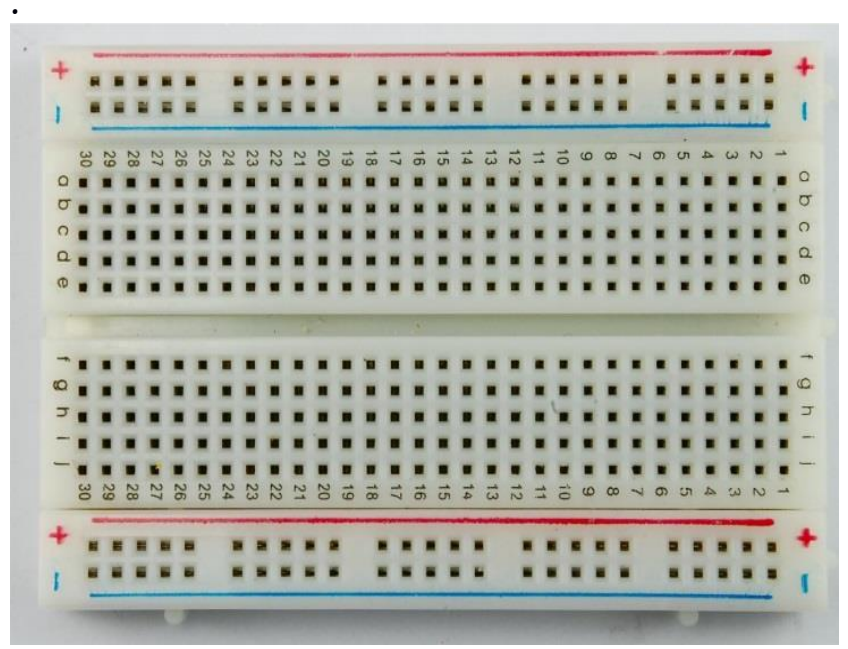

Figure 5: Breadboard from Arduino

B. Software Components

1) Coding Platform: Arduino
Arduino IDE is an open source software that is can accepts many program languages as JAVA, Python, $\mathrm{C}, \mathrm{C}++$. It allows the user to write code in a special environment with syntax highlighting and other features which makes writing a code, an easy process. After writing the code, it is loaded in the $\mathrm{I} / \mathrm{O}$ board [9].

\section{2) Coding Language: $C$}

$C$ is procedural programming language developed by Dennis Ritchie in 1972. This language was developed as a programming language to write operating system. Its features were derived from an earlier language called "B". It is one of the most used and powerful languages because it is reliable, flexible, efficient and effective.

3) Interface Builder: Blynk

Blynk is a platform that allows the user to design interfaces to control and monitor the hardware projects. With Blynk we can create a project dashboard and arrange buttons, graphs, maps and widgets on the screen. With these widgets, pins can be turned in and off and display data from sensors [10].

\section{Results}

Table 1 shows result of different smartwatch to care about the safety of the users. These smartwatches have brought a lot of technology and they are set at higher price which not everyone can afford it. Out of that it's required a smart phone to be connected via Bluetooth.

TABLE I

, Existing Smart Watch Comparison

\begin{tabular}{|c|l|}
\hline \multicolumn{1}{|c|}{ Name } & \multicolumn{1}{c|}{ Technology } \\
\hline Apple Watch 6 & $\begin{array}{l}\text { Blood Oxygen sensor, Heart Rate, } \\
\text { Sleep time control, Fitness, } \\
\text { Motivation, GPS, Cellular Network, } \\
\text { Bluetooth5, Message and } \\
\text { Notification }\end{array}$ \\
\hline Galaxy Watch 3 & $\begin{array}{l}\text { Oxygen Ratting, Fall down } \\
\text { Detection, Night Sleep control, } \\
\text { Improve every stride, Bluetooth5, } \\
\text { Message and Notification }\end{array}$ \\
\hline Huawei watch GT & $\begin{array}{l}\text { Long battery life, Real time Heart } \\
\text { Rate monitoring, Connected with } \\
\text { 3 Satellites and 1 precise location, } \\
\text { Intelligent sleep statistics, } \\
\text { Bluetooth5 Message and } \\
\text { Notification }\end{array}$ \\
\hline
\end{tabular}

All the hardware components cited previously have been connected and work as a prototype of the smartwatch. The 
prototype has a heart rate sensor, a GPS tracking functionality and a push button that works as a panic button.

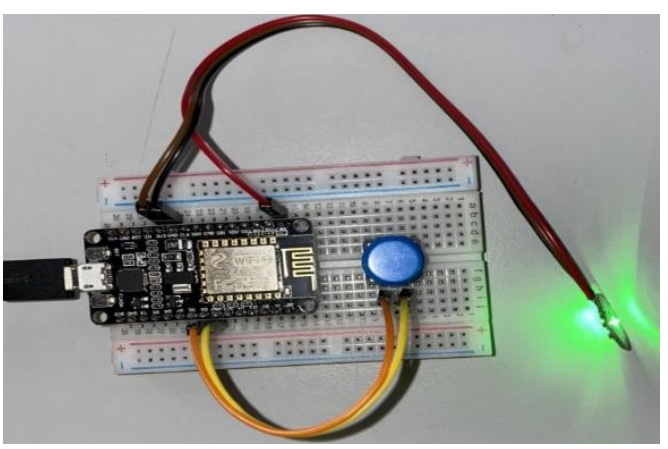

Fig. 6 Prototype of the Smart Watch

When the user touches the heart rate sensor shown above, the Wi-Fi module sends the heartbeat rate to the mobile application. Furthermore, it sends the real time location of the user to the application and it sends a notification when the button is pushed.

\section{Software}

To design our software, we used a platform called Blynk. Fig. 7: After the emergency was declared, we proceed with the rescue. The application tracks the device and displays both location of the smartwatch user and location of the rescue team.

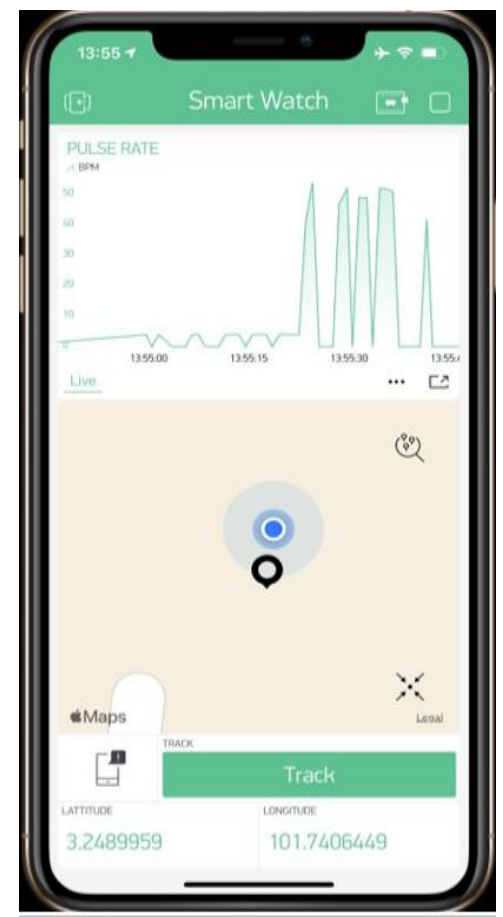

Fig. 7: After the emergency was declared, we proceed with the rescue. The application tracks the device and displays both location of the smartwatch user and location of the rescue team
When the device is connected to the satellite, it is shown in our map (Fig. 7). The smartwatch will automatically keep sending heartbeat information (Fig. 7). When the user clicks the SOS button, an "Emergency Detected" (Fig. 7) will notify the admin. The admin will track user's location and send it to the rescue team. They will proceed with the rescue (Fig. 7).

\section{CONCLUSION}

The project "Smartwatch for fishermen" has been designed in order to provide a form of security and help in case of emergency. The fishermen wear the watch to send his location and heartbeat rate to the rescue team so they can come and save him if an accident happen. There are numerous difficulties that have been faced in this project as well as positive and negative feedback from the testers. Those comments were essential in making our project better than expected. Thankfully we have been able to create a prototype of the smartwatch as well as a software that displays the information recorded by the smartwatch. Hopefully this project will be put in use soon and will provide more security for those in need.

\section{REFERENCES}

[1] A. Kuparinen A, Boit A, Valdovinos FS, Lassaux $H$, Martinez ND. Fishing-induced life-history changes degrade and destabilize harvested ecosystems. Sci Rep [Internet].;6(1):22245. Available from: https://doi.org/10.1038/srep22245. 2016

[2] O.F. Science OF. (12) Patent Application Publication (10) Pub. No: US 2016 / 0271610 A1 Patent Application Publication. 2016;1(19).

[3] M.K. Urgen MK. Patent Application Publication (10) Pub. No: US 2010 / 0224498A1.;1(19).2011

[4] Mccaffrey. ( 12 ) United States Patent.;2(12). 2019

[5] J. Martinez, "Tracking and safety Smartwatch", U.S. Patent 9747771 B1, August 29, 201

[6] D.S. Quintana Alvares GA, Heathers JAJ. Guidelines for Reporting Articles on Psychiatry and Heart rate variability (GRAPH): recommendations to advance research communication. Transl Psychiatry [Internet].

[7] H. Lee Tak J, Choi J. Wearable Antenna Integrated into Military Berets for Indoor/Outdoor Positioning System. IEEE Antennas Wirel Propag Lett.;16:1919-22. 2017

[8] K. Inso Noicharoen P, Meathatanunchai N, Khurat A. Play it safe a personal security application on Android platform. In: 2016 Fifth ICT International Student Project Conference (ICT-ISPC). p. 133-6. 2016

[9] S. Arakliotis, LAWRIS: A Rule-Based Arduino Programming System for Young Students. 3, 3-6. 2016

[10] H.S. Doshi, INTERNET of THINGS ( IOT ): INTEGRATION of BLYNK for DOMESTIC USABILITY. 1(4), 149-157. 2017 